\title{
Bilateral Hydronephrosis as a Consequence of Vesicoureteral Reflux in a 6-Year-Old Male Patient
}

\author{
Enrique Konstat-Korzenny* (iD and Raquel Cohen-Shaooli (iD
}

Instituto de Salud del Estado de México, México

*Corresponding author: Enrique Konstat-Korzenny, Instituto de Salud del Estado de México, Fuente de Las pirámides 16depot 103, México, Tel: 5530387366

\begin{abstract}
Vesicoureteral reflux (VUR) is the most common urologic pathology in the pediatric population. VUR is defined as primary when the intravesical portion of the ureters is short and produces an incompetent ureterovesical junction. Secondary VUR is caused by anatomical or functional obstruction to bladder emptying, which in turn increases the bladder internal pressure, therefore overcoming the ureterovesical junction and causing reflux. Current guidelines reflect on whether patients of a certain age with a febrile urinary tract infection should be offered imaging studies to detect urinary tract anatomical abnormalities, but there are conflicting recommendations for other age groups. VUR should be a high suspicion on patients with recurrent urinary tract infections, and it should be detected early to prevent further renal damage that can lead to end-stage renal disease.
\end{abstract}

\section{Introduction}

In this work we present the case of a 6-year-old male patient who presented to a rural clinic in Mexico, where after a thorough medical work up, we found severe bilateral hydronephrosis caused by vesicoureteral reflux. We also provide an analysis of the pathology with the current guideline recommendations.

\section{Case Presentation}

A 6-year-old male presents to an outpatient rural clinic in the State of Mexico, Mexico. The mother was concerned about his failure to thrive and two "little masses" that appeared in his inguinal region and neck. The mother noticed them four days previously, and described them as mobile, and non-painful. She endorsed symptoms consistent with an upper respiratory tract infection, without fever. She also mentioned that her child has had approximately four episodes of self-resolving epistaxis in the previous 2 to 3 months. She denied any bone pain, night fevers or diaphoresis. Of note, neither the mother nor the child endorsed any urinary symptoms or abnormal voiding patterns. The patient was not taking any medications, he had no known drug allergies. Surgical and perinatal history was negative for any pathologies, and he reached all developmental milestones according to his age.

Physical exam revealed a well-appearing child, with the following vital signs; $36.6^{\circ} \mathrm{C}$ of temperature, weight of $17.5 \mathrm{Kg}\left(5^{\text {th }}\right.$ percentile) and a height of $108 \mathrm{~cm}\left(25^{\text {th }}\right.$ percentile). Relevant physical examination showed an erythematous pharynx without exudate, and hyaline rhinorrhea. Inguinal nodes were felt bilaterally, mobile, without any pain to palpation. The rest of the physical examination was noncontributory. The patient was treated symptomatically with acetaminophen and loratadine for 5 days for the respiratory symptoms. Because of the mother's concerns of epistaxis and failure to thrive, general blood and urine exams were ordered.

Four days later, the patient returned to the clinic. The mother stated that the child was feeling better and currently asymptomatic. CBC revealed Leukocytes 5.23 $10^{3} / \mu \mathrm{L}, \mathrm{Hb} 13.3 \mathrm{~g} / \mathrm{dL}$, Hematocrit 42.8\%, MCV $77.1 \mathrm{fL}$, Platelets $37610^{3} / \mu \mathrm{L}$, Glucose $87 \mathrm{mg} / \mathrm{dL}$, Urea $42.9 \mathrm{mg} /$ $\mathrm{dL}$, BUN $20 \mathrm{mg} / \mathrm{dL}$, Creatinine $0.56 \mathrm{mg} / \mathrm{dL}$.

Urinalysis demonstrated Specific Gravity 1.018, pH 8.00, Leukocyte esterase 500/ $\mu \mathrm{L}$, Positive nitrites, Protein $25 \mathrm{mg} / \mathrm{dL}$, negative for glucose and ketones. Microscopic exam revealed Leukocytes $8 /$ field, no eryth-

Citation: Korzenny EK, Shaooli RC (2021) Bilateral Hydronephrosis as a Consequence of Vesicoureteral Reflux in a 6-Year-Old Male Patient. Clin Med Rev Case Rep 8:350. doi.org/10.23937/2378$3656 / 1410350$

Accepted: May 29, 2021: Published: May 31, 2021

Copyright: (c) 2021 Konstat-Korzenny E, et al. This is an open-access article distributed under the terms of the Creative Commons Attribution License, which permits unrestricted use, distribution, and reproduction in any medium, provided the original author and source are credited. 
rocytes, abundant bacteria, no epithelial or squamous cells and no casts. Other serum tests were within normal limits. The patient and the mother still did not endorse any urinary symptoms.

Because of the urinalysis suggesting a urinary tract infection in a male patient and the low weight, a kidney, ureter and bladder ultrasound was ordered to look for any anatomical or structural abnormalities (Figure 1). Further, before the vesicoureteral reflux diagnosis was clear, the patient was treated for a presumed urinary tract infection with PO amoxicillin/clavulanate for 5 days.
The ultrasound reported bilateral hydronephrosis. The right kidney measured $59 \times 33 \times 35 \mathrm{~mm}$, with a volume of $37.1 \mathrm{ml}$, with loss of the sinus-parenchymal relation due to collecting system dilation extending to the renal pelvis and chalices, compressing the renal parenchyma.

The left kidney measured $72 \times 35 \times 32 \mathrm{~mm}$, a volume of $72.8 \mathrm{ml}$, with preserved medulla-cortex and sinus-parenchymal relation. Cortex measured $6 \mathrm{~mm}$. No evidence of collecting system dilation was reported. The bladder was described in its usual form and location,
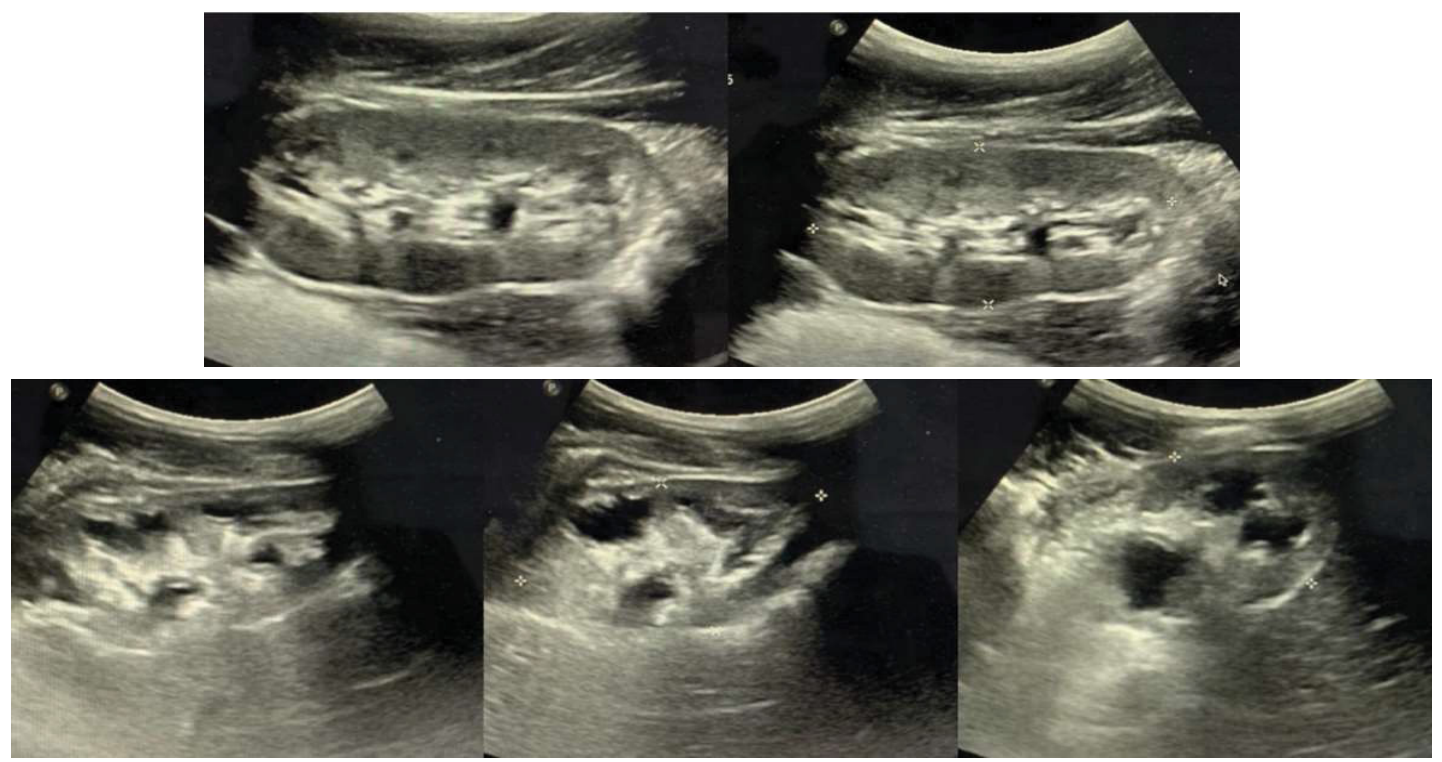

Figure 1: Kidney ultrasound demonstrating hydronephrosis and collecting system dilation of the right kidney (bottom images). The left kidney (top images), was also reported with hydronephrosis but does not show collecting system dilation.

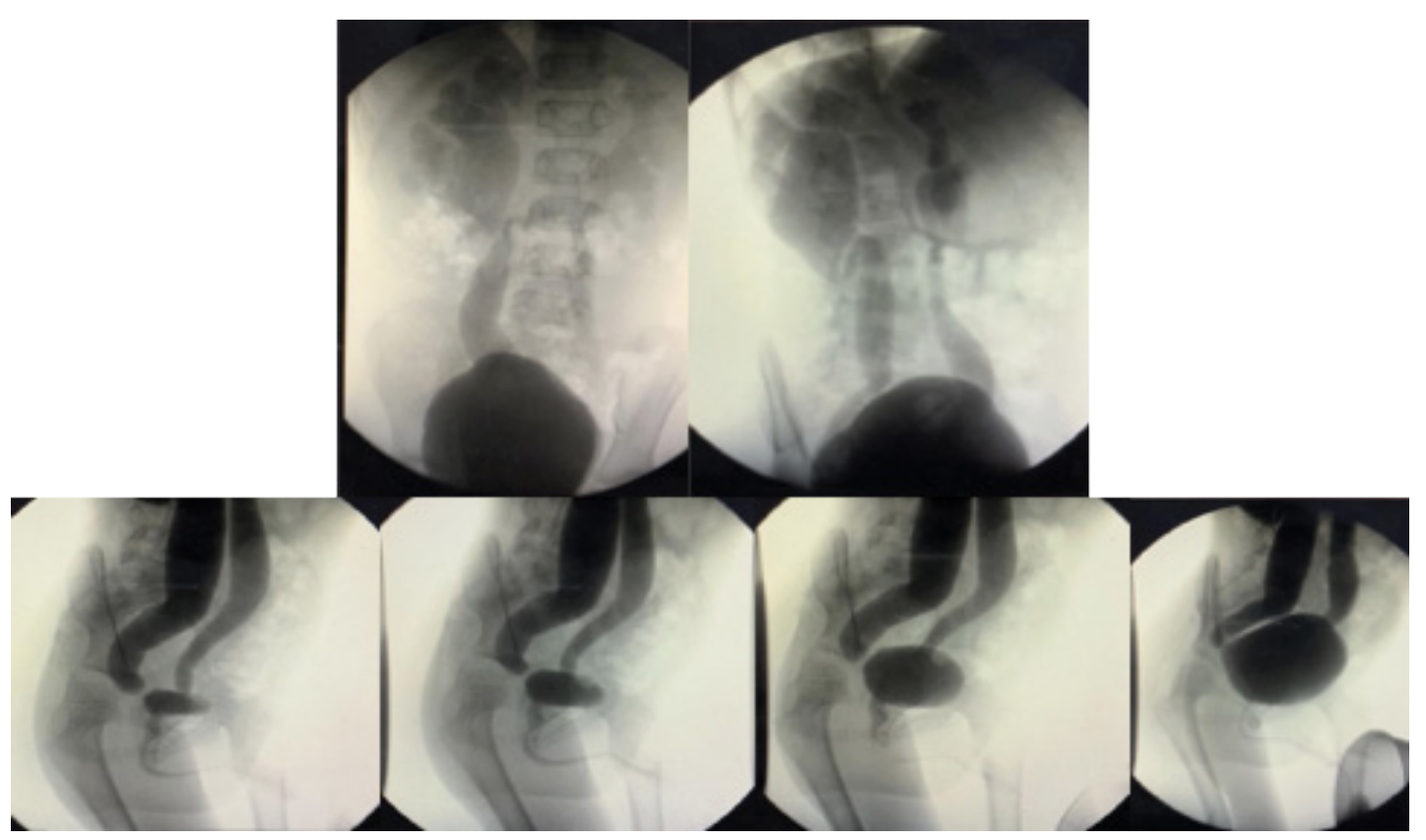

Figure 2: Voiding cystourethrography demonstrated bilateral ureteral dilation and tortuosity with right predominance, the right kidney shows dilation of pelvis and calyces. 
with regular, defined borders, a $276 \mathrm{ml}$ volume and 1 $\mathrm{mm}$ thickness. Postmictional volume was $110.2 \mathrm{ml}$, with a $39.9 \%$ of urinary residual volume. Bilateral ureteral dilation, with right side predominance was reported.

With these ultrasonographic findings, a voiding cystourethrography (VCUG) was performed, in which ureteric and pelvicalyceal dilation was evident and the diagnosis of Grade V VUR in the right kidney and Grade IV VUR in the left kidney were confirmed (Figure 2). The patient was eventually referred to a pediatric urologist for further surgical management.

\section{Discussion}

Normally, the ureters enter the bladder at the ureterovesical junction, and a segment of them lies inside the bladder (intravesical ureter). Reflux is prevented by contraction of the bladder and the surrounding muscles. Vesicoureteral reflux (VUR) is a urologic pathology characterized by retrograde passage of urine from the bladder to the upper urinary tract (ureters and kidneys).

VUR is the most common urologic pathology in the pediatric population. Epidemiological studies report an estimated prevalence of $1 \%$ of all neonates $[1,2]$. The prevalence increases in cases where hydronephrosis is diagnosed prenatally and in patients with febrile urinary tract infections (UTI) [3,4].

VUR is defined as primary when the intravesical portion of the ureters is short and produces an incompetent ureterovesical junction. Most of these cases are congenital and have a strong genetic component $[5,6]$. Secondary VUR is caused by anatomical or functional obstruction to bladder emptying, which in turn increases the bladder internal pressure, therefore overcoming the ureterovesical junction and causing the reflux. Examples of these are posterior urethral valves, neurogenic bladder and prostate pathologies. In our presented case, the child was diagnosed with primary VUR, as no obstructive or bladder pathologies were found in the mother's pregnancy ultrasound reports or during his previous visits.

The clinical course of VUR is varied and sometimes asymptomatic. Most cases are diagnosed when hydronephrosis is found during prenatal ultrasonography. Postnatally, urinary symptoms may be evident such as enuresis, tenesmus or abnormal voiding patterns, how- ever, patients mainly present with recurrent febrile urinary tract infections (UTI) [7]. It is worth noting that our patient was completely asymptomatic, even in an advanced stage of the pathology.

Although there is not a consensus on current international guidelines, an abdominal or kidney/bladder ultrasound is recommended for all patients younger than two years old with a febrile UTI and risk factors such as family history of VUR, septicemia, renal insufficiency, non-responsive UTI after 72 hours of antibiotics and UTI due to bacteria different than E. coli [8]. Ultrasonography may be a cost-effective study; however, it may not detect renal scarring or mild to moderate cases of VUR where no ureteral dilation is present [9]. Even so, the AAP recommends performing it, since the study identifies most of the grades IV and V VUR, which present a major risk for renal scarring. While it does not usually identify lower grades, these grades are not associated with renal damage $[10,11]$.

While initially an ultrasound may be useful to detect the complications of the pathology, the definite diagnosis is made through a voiding cystourethrography (VCUG) or radionuclide cystogram. VCUG is a fluoroscopic imaging study that provides images of the upper urinary tract. This study is the gold-standard test to diagnose VUR [12]. The disadvantages of this study are high gonadal radiation and intermittent imaging. Other methods such as Direct Radionuclide Cystography (DRC) and Indirect Radionuclide Cystography are used for evaluation of VUR, with less radiation and increased sensitivity. In the first method, the radionuclide is introduced via a catheter in the urethra while the latter avoids the urethral catheterization $[13,14]$.

To assess renal scarring, a technetium-99m-labeled dimercaptosuccinic acid renal imaging study can be performed. The American Urological Association recommends performing this study, especially to children with grades III-V VUR, since those are the stages that most likely present with renal scarring. This study can provide useful information in the decision-making process, as well as a baseline information for a comparison once the VUR has spontaneously or surgically resolved [15].

In 1981, the International Reflux Study Committee published a 5-stage grading system for this pathology, which is still presently used. It classifies VUR according

Table 1: Vesicoureteral reflux grades according to the international reflux study committee.

\section{GRADES ANATOMICAL LEVEL AND AFFECTED STRUCTURES}

\begin{tabular}{|l|l|}
\hline I & Reflux is limited to the ureter (no ureter dilation) \\
\hline II & Reflux fills the ureter, renal pelvis and collecting system (no dilation) \\
\hline III & $\begin{array}{l}\text { Reflux fills the ureter, renal pelvis and collecting system (Mild ureteral and pelvicalyceal dilation) } \\
\text { Reflux fills the ureter, renal pelvis and collecting system (Gross ureteral dilation with mild tortuosity and calyceal } \\
\text { blunting) }\end{array}$ \\
\hline V & $\begin{array}{l}\text { Reflux fills the ureter, renal pelvis and collecting system (Severe ureteral dilation and tortuosity, severe calyceal } \\
\text { blunting, loss of papillary impressions and fornices, intrarenal reflux) }\end{array}$ \\
\hline
\end{tabular}




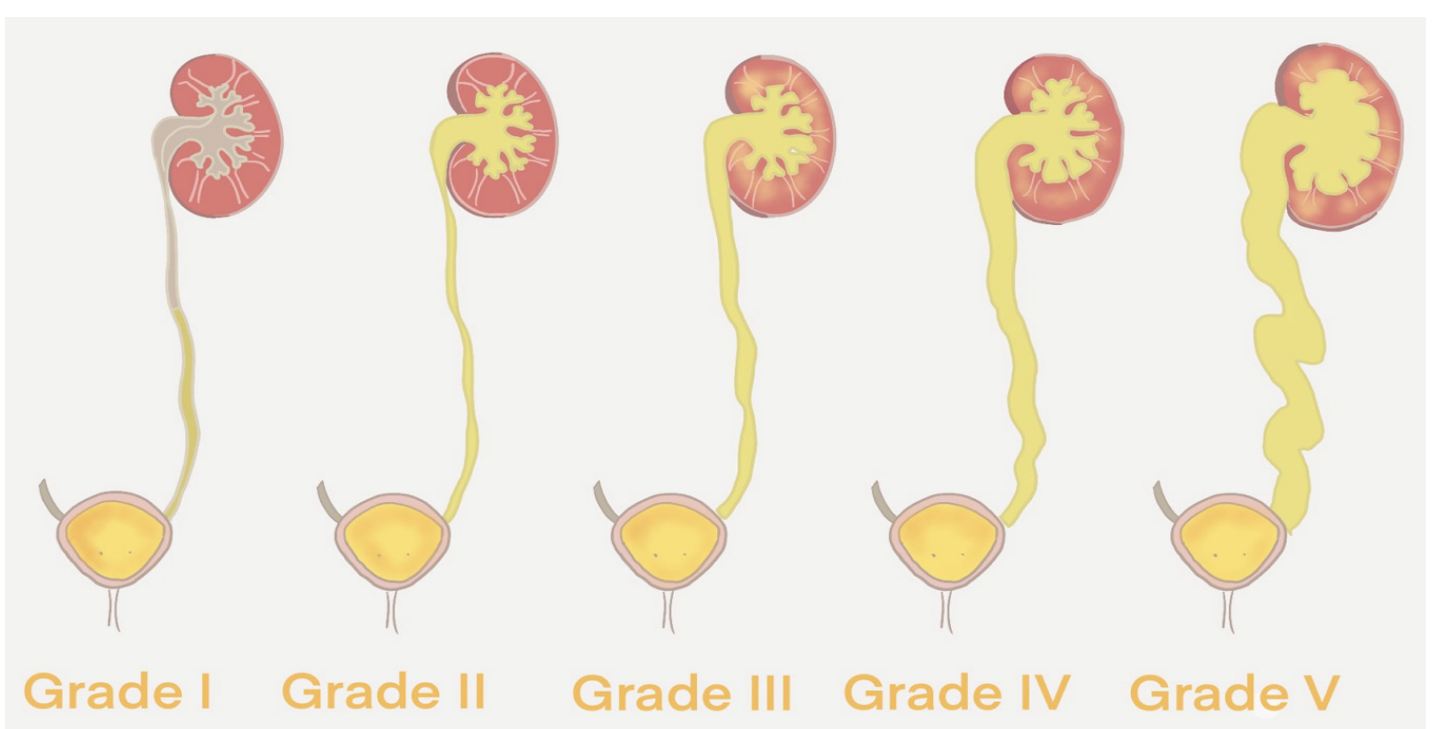

Figure 3: Schematic classification of vesicoureteral reflux.

to the anatomic level of the affection and renal collecting system dilation assessed in the VCUG [16] (Table 1 and Figure 3).

Prognosis of this pathology depends on the grade of the reflux and if it is primary or secondary VUR. Primary VUR tends to improve with age, as the intravesical portion of the ureter grows and develops a better anti-reflux mechanism. The lower the grade, the better the prognosis of the pathology. Grades I and II VUR may have spontaneous resolution by 5 years of age in up to $80 \%$ of the patients [17]. In those cases, the American Urological Association recommends yearly follow-up with general evaluations (blood pressure, height, weight), annual urinalysis looking for proteinuria and bacteriuria, and annual kidney ultrasonography to monitor renal growth and parenchymal scarring [18].

Higher grades will usually not resolve on their own and will benefit from surgical interventions as well as daily antibiotic prophylaxis. The rationale for treatment is to prevent further anatomic damage to the kidneys, as well as episodes of pyelonephritis and renal scarring which could eventually lead to end-stage renal disease. Since VUR predisposes to UTIs, daily prophylactic antibiotics are prescribed for higher grades of VUR. Although recent studies have found conflicting evidence to support this practice, most guidelines still recommend it $[15,19]$. The definite treatment for VUR is strictly surgical. Different techniques are described, both open, endoscopic or robotic surgery, but their description is beyond the scope of this work.

It is worth mentioning that according to the American Urological Guidelines, the prevalence of VUR in siblings of affected children is $27 \%$. Once the diagnosis of our patient was established, we proposed the mother an observational approach for her other younger sons, with the necessity of a kidney and bladder ultrasound if any sibling presented with signs of a UTI or failure to thrive [15].
In the case presented, we believe that the patient's urinary laboratory findings were due to urinary stasis secondary to VUR, which is a predisposing factor for febrile UTI. Even though the patient was completely asymptomatic regarding urinary symptoms, the presence of urinary laboratory findings and the failure to thrive made a kidney etiology probable, after all the other work up proved to be normal.

\section{Conclusion}

Congenital urological abnormalities and vesicoureteral reflux are diagnoses to keep in mind in children with recurrent UTIs, especially in male patients. In our case, an initial non-febrile urinary tract infection in a 6-yearold male, had we followed the current guidelines and not order ultrasonography, we would not have found the hydronephrosis. Early detection is critical to prevent long term complications such as end-stage renal disease. Unfortunately, in our rural setting, full guideline interventions are not readily available, therefore, only a kidney and bladder ultrasound were initially performed as it is a readily available and cost-effective study, and once the structural damage was identified, VCUG was performed in which the VUR was confirmed.

\section{Conflicts of Interest}

The authors declare no conflict of interest.

\section{References}

1. Hiraoka $M$, Hori $C$, Tsukahara $H$, Kasuga $K$, Ishihara $Y$, et al. (1999) Vesicoureteral reflux in male and female neonates as detected by voiding ultrasonography. Kidney Int 55: 1486-1490.

2. Arant BS (1991) Vesicoureteric reflux and renal injury. Am J Kidney Dis 17: 491-511.

3. Hoberman A, Charron M, Hickey RW, Baskin M, Kearney $\mathrm{DH}$, et al. (2003) Imaging studies after a first febrile urinary tract infection in young children. N Engl J Med 348: 195202. 
4. Cosentino M, Caffaratti J, Bujons A, Garat JM, Villavicencio H (2013) Vesico-ureteral reflux, endoscopic management. Arch Argent Pediatr 111: 349-352.

5. Yosypiv IV (2012) Congenital anomalies of the kidney and urinary tract: A genetic disorder? Int J Nephrol 2012: 909083.

6. Chen F (2009) Genetic and developmental basis for urinary tract obstruction. Pediatr Nephrol 24: 1621-1632.

7. Safdar O, Safhi MA, Saggaf O, Algethami HR, Alhalabi M, et al. (2020) Assessment of the etiologies and outcomes of antenatal hydronephrosis in patients at king abdulaziz university hospital. Cureus 12: e7615.

8. Okarska-Napierała M, Wasilewska A, Kuchar E (2017) Urinary tract infection in children: Diagnosis, treatment, imaging-comparison of current guidelines. J Pediatr Urol 13 : 567-573.

9. Massanyi EZ, Preece J, Gupta A, Lin SM, Wang MH (2013) Utility of screening ultrasound after first febrile UTI among patients with clinically significant vesicoureteral reflux. Urology 82: 905-909.

10. Subcommittee on urinary tract infection, steering committee on quality improvement and management, Roberts KB (2011) Urinary tract infection: Clinical practice guideline for the diagnosis and management of the initial UTI in febrile infants and children 2 to 24 months. Pediatrics 128: 595.

11. American Academy of Pediatrics (2016) Reaffirmation of AAP clinical practice guideline: The diagnosis and management of the initial urinary tract infection in febrile infants and young children 2-24 months of age. Pediatrics 138: e20163026.

12. Lebowitz RL, Olbing H, Parkkulainen KV, Smellie JM, Tam-
minen-Mobius TE (1985) International system of radiographic grading of vesicoureteric reflux. International reflux study in children. Pediatr Radiol 15: 105-109.

13. Unver T, Alpay H, Biyikli NK, Ones T (2006) Comparison of direct radionuclide cystography and voiding cystourethrography in detecting vesicoureteral reflux. Pediatr Int 48: 287-291.

14. Gordon I, Peters AM, Morony S (1990) Indirect radionuclide cystography: A sensitive technique for the detection of vesico-ureteral reflux. Pediatr Nephrol 4: 604-606.

15. American Urological Association (2010) Management and screening of primary vesicoureteral reflux in children.

16. (1981) Medical versus surgical treatment of primary vesicoureteral reflux: Report of the international reflux study committee. Pediatrics 67: 392-400.

17. Elder JS, Peters CA, Arant Jr BS, Ewalt DH, Hawtrey CE, et al. (1997) Pediatric vesicoureteral reflux guidelines panel summary report on the management of primary vesicoureteral reflux in children. J Urol 157: 1846-1851.

18. Skoog SJ, Peters CA, Arant Jr BS, Copp HL, Elder JS, et al. (2010) Pediatric vesicoureteral reflux guidelines panel summary report: Clinical practice guidelines for screening siblings of children with vesicoureteral reflux and neonates/infants with prenatal hydronephrosis. J Urol 184: 1145-1151.

19. Wong NC, Koyle MA, Braga LH (2017) Continuous antibiotic prophylaxis in the setting of prenatal hydronephrosis and vesicoureteral reflux. Can Urol Assoc J 11: S20-S24. 